\title{
COMPARAÇÃO DO ÍNDICE DE TORG OBTIDO POR MEIO DE RADIOGRAFIA E RESSONÂNCIA MAGNÉTICA NOS PACIENTES COM MIELOPATIA CERVICAL ESPONDILÓTICA
}

\author{
COMPARISON OF THE TORG INDEX OBTAINED BY RADIOGRAPHY AND MAGNETIC RESONANCE \\ IN PATIENTS WITH SPONDYLOTIC CERVICAL MYELOPATHY
}

COMPARACIÓN DEL ÍNDICE DE TORG, OBTENIDO POR MEDIO DE RADIOGRAFÍA Y RESONANCIA, EN LOS PACIENTES CON MIELOPATÍA ESPONDILÓTICA CERVICAL

Alexandre de Oliveira Zam¹, Priscila Rossi de Batista², Diogo Miranda Barbosa ${ }^{3}$, Jose lucas Batista Junior ${ }^{4}$, Charbel Jacob Junior ${ }^{4}$, igor Cardoso Machado ${ }^{4}$, Rodrigo Rezende ${ }^{5}$

\begin{abstract}
RESUMO
Objetivo: A Mielopatia Cervical Espondilótica (MCE) é uma disfunção da medula espinhal relacionada à degeneração típica do envelhecimento. No estudo de imagem podemos obter a medida do Índice de Torg, para estimar a estenose cervical. Objetivamos, assim, medir o Índice de Torg através de radiografia e ressonância magnética (RM), possibilitando verificar possíveis discrepâncias entre os métodos de medida. Métodos: Realizada mensuração do Índice de Torg na radiografia e na RM da coluna cervical, sendo obtido através da relação entre a superfície posterior do corpo vertebral e o ponto mais próximo à linha laminar correspondente, dividido pelo diâmetro sagital do corpo vertebral. Resultados: Participaram 29 pacientes, sendo 10 mulheres e 19 homens, com médias de idade 48,1 \pm 11 anos, de peso $68,7 \pm 5 \mathrm{Kg}$ e de altura 1,68 \pm 0,6 $\mathrm{m}$. Houve diferença significativa entre o Índice de Torg calculado através de radiografia e RM, sendo menores os índices observados na RM (radiografia: 0,73 $\pm 0,17$ vs. RM: 0,48 $\pm 0,14, p<0,05$ ). No entanto, ambas as aferições traduzem o mesmo resultado: estenose cervical absoluta (Índice de Torg $<0,8$ ). Conclusões: Nossos resultados corroboram relatos de outros autores que acreditam que o Índice de Torg medido através da radiografia, como preconizado, minimiza a real estenose do canal cervical. Sugerimos em nosso estudo que a RM permite melhor estimativa do grau de estenose do canal, muito embora nossos resultados em relação ao grau de estenose cervical tenham sido semelhantes estatisticamente.
\end{abstract}

Descritores: Coluna vertebral; Doenças da coluna vertebral; Radiologia; Radiografia; Imagem por ressonância magnética.

\begin{abstract}
Objective: Cervical spondylotic myelopathy (SCM) is a dysfunction related to spinal cord degeneration typical of aging. In the imaging studies can achieve a measure of the Torg index to estimate cervical stenosis. We aim therefore to measure the Torg index in X-rays and Magnetic Resonance (MR), to investigate possible discrepancies between the methods of measurement. Methods: The Torg Index was measured on radiographs and MR of the cervical spine, obtained by determining the relationship between the posterior surface of the vertebral body and the nearest point to the corresponding laminar line, divided by the sagittal diameter of vertebral body. Results: A total 29 patients has participated, 10 women and 19 men, with mean age of $48.1 \pm 11$ years, weight $68.7 \pm 5 \mathrm{~kg}$ and height $1.68 \pm 0.6 \mathrm{~m}$. There were significant differences between the Torg Index calculated by radiography and MR, and lower rates observed on MR (radiography: $0.73 \pm 0.17$ vs. RM: $0.48 \pm 0.14, p<0.05)$. However, both measurements reflect the same result: absolute cervical stenosis (Torg index < 0.8). Conclusions: Our results confirm reports by other authors who believe that the Torg Index measured by radiography, as recommended, minimizes the actual cervical canal stenosis. Our study suggests that MRI allows better estimate of the degree of stenosis, although our results regarding the degree of cervical stenosis were statistically similar.
\end{abstract}

Keywords: Spine; Spinal diseases; Radiology; Radiography; Magnetic resonance imaging.

\section{RESUMEN}

Objetivo: La Mielopatía Cervical Espondilótica (MCE) es un trastorno relacionado con la degeneración de la médula espinal, típica del envejecimiento. En el estudio de imagen se puede obtener la medida del Índice de Torg para estimar la estenosis cervical. Nuestro objetivo es, por tanto, medir el índice de Torg mediante rayos-X y resonancia magnética (RM), posibilitando verificar posibles discrepancias entre los métodos de medición. Métodos: Se realiza la medición del Índice de Torg en la radiografía y en la resonancia magnética de la columna cervical, siendo obtenido mediante la determinación de la relación entre la superficie posterior del cuerpo vertebral y el punto más cercano a la línea de la lámina correspondiente, dividido por el diámetro sagital del cuerpo vertebral. Resultados: Participó un total de pacientes 29, siendo 10 mujeres y 19 hombres, edad promedio de 48,1 \pm 11 años, peso 68,7 $\pm 5 \mathrm{Kg}$ y altura de 1,68 \pm 0,6 $\mathrm{m}$. No hubo diferencias significativas entre el Índice de Torg calculado por la radiografía y el de la resonancia magnética, siendo menores los índices observados en la RM (radiografía: 0,73 $\pm 0,17$ vs $R M$ : 0,48 $\pm 0,14, p<0,05$ ). Sin embargo, ambas mediciones reflejan el mismo resultado: estenosis

1. Ortopedista eTraumatologista Especialista em Cirurgia da Coluna Vertebral pelo Grupo de Coluna da Santa Casa de Vitória - Vitória, ES, Brasil.

2. Fisioterapeuta assistente do Grupo de Coluna Vertebral da Santa Casa de Vitória - Vitória, ES, Brasil.

3. Radiologista Especialista em Sistema Músculo-esquelético, Chefe da Residência em Radiologia do Centro de Diagnóstico por Imagem (CDI) - Vitória, ES, Brasil.

4. Ortopedista eTraumatologista Especialista em Cirurgia da Coluna Vertebral, Médico assistente do Grupo de Coluna Vertebral da Santa Casa de Vitória e do Vila Velha Hospital-Vitória, ES, Brasil.

5. Ortopedista eTraumatologista Especialista em Cirurgia da Coluna Vertebral, Chefe do Grupo de Coluna Vertebral da Santa Casa de Vitória e do Vila Velha Hospital - Doutor em Ciências da Saúde.

Trabalho realizado no Hospital Meridional (Cariacica, ES), Vila Velha Hospital (Vila Velha, ES) e Hospital Santa Casa de Misericórdia de Vitória (Vitória, ES).

Correspondência: Rodrigo Rezende. Rua Desembargador Augusto Botelho, 209/801 - 29101-110 - Praia da Costa, Vila Velha - ES, Brasil. grupodecoluna @ santacasavitoria.org

Recebido em 20/03/2011, aceito em 03/08/2011 
cervical absoluta (Índice de Torg <0,8). Conclusiones: Nuestros resultados confirman los informes de otros autores quienes creen que el Índice de Torg medido por radiografía, como se recomienda, minimiza la estenosis real del canal cervical. En nuestro estudio se sugiere que la RM permite hacer una mejor estimativa del grado de estenosis del canal, aunque nuestros resultados, sobre el grado de estenosis cervical, fueron estadísticamente similares.

Descriptores: Columna vertebral; Enfermedades de la columna vertebral; Radiología; Radiografía; Imagen por resonancia magnética.

\section{INTRODUÇÃO}

A Mielopatia Cervical consiste em uma disfunção da medula espinhal na qual ocorre um processo de desmielinização das células do tecido nervoso central. Diversas etiologias são responsáveis pela doença como defeitos congênitos, trauma, doença degenerativa, doenças inflamatórias e infecciosas assim como doenças metabólicas ${ }^{1}$. Nosso estudo avalia somente a Mielopatia Cervical Espondilótica (MCE) devido a alterações congênitas e degenerativas típicas do envelhecimento, cuja fisiopatologia primária se relaciona a uma compressão da medula espinhal cervical $^{2,3}$, com consequente isquemia e morte de células do tecido nervoso central.

A prevalência exata é desconhecida, porém, sabe-se que é mais comum em pacientes acima de 55 anos $^{4,5}$, do sexo masculino, numa proporção de $3: 2^{3}$, sendo a localização mais frequente da estenose na transição entre o nível cervical C5-C6 6 ${ }^{3,6}$. Há maior predisposição nos pacientes da raça Oriental ${ }^{7,8}$ devido prevalente ossificação do ligamento longitudinal posterior, que contribui para um aumento da estenose.

No quadro clínico da MCE encontramos distúrbios da função motora, sensitiva e dos reflexos profundos. São frequentes as alterações da função dos movimentos finos da extremidade superior, fadiga precoce em movimentos repetitivos e perda da coordenação motora, o distúrbio da marcha é frequentemente a primeira manifestação clinica. Perda do controle vesical e intestinal podem ocorrer em 20 a 50\% dos pacientes ${ }^{3,9,10}$, entretanto, é uma manifestação tardia da doença.

Na radiografia da coluna cervical na incidência ântero-posterior (AP) é possível visualizar estreitamento do espaço unco-vertebral ${ }^{2}$, enquanto na incidência em perfil observa-se estreitamento do canal vertebral, diminuição do espaço discal, presença de osteófitos, perda do alinhamento no plano sagital com perda da lordose cervical fisiológica ou espondilósteses².

A partir da radiografia em perfil, Torg e Pavlov criaram um método para aferir a estenose do canal cervical, com o objetivo de eliminar o viés da medida feita através de mensuração direta do canal vertebral, que era considerada normal quando acima de $13 \mathrm{~mm}^{11}$.

Na mensuração direta do canal cervical a magnificação radiológica provocada ${ }^{12,13}$ pela variação da distância entre o paciente, o filme e o tubo de radiação, proporciona uma medida que varia entre diferentes examinadores. Torg e Pavlov propuseram uma relação entre o canal vertebral, sobre o corpo vertebral correspondente. Essa medida ficou conhecida como Índice de Torg, Índice de Pavlov ou ainda Índice de Torg e Pavlov. Este método trouxe grande avanço no diagnóstico precoce da MCE e é atualmente usado por diversas escolas médicas em todo o mundo 3 , 8,11,13-20

No entanto, alguns autores ${ }^{21,22}$ relatam que o índice de Torg não esta necessariamente associado a estenose cervical devido à variabilidade do tamanho do corpo vertebral. Outro viés ocorre pois o índice é medido no meio do corpo vertebral, e as principais causas da estenose cervical que são: as formações osteofitárias, espessamentos ligamentare ${ }^{15}$ e hérnias discais ${ }^{3,23,24}$, geralmente ocorrem em torno do disco intervertebral. Isso contribui para uma perda da sensibilidade diagnóstica do processo patológico em questão.

Devido à existência de dúvidas na literatura mundial sobre a possibilidade do Índice de Torg aferido na radiografia minimizar a real estenose do canal vertebral cervical, objetivamos em nosso estudo comparar o Índice de Torg obtido através de radiografia e de ressonância magnética da coluna cervical.

\section{MÉTODOS}

Foram avaliados pacientes de ambos os sexos que seriam submetidos a tratamento cirúrgico de MCE, durante o período de 2009 e 2010, como critérios de exclusão do trabalho foram todos os pacientes com mielopatia de causas não degenerativas.

O Índice de Torg e Pavlov foi obtido através da relação entre a superfície posterior do corpo vertebral e o ponto mais próximo à linha laminar correspondente, dividido pelo diâmetro sagital do corpo vertebral, como esquematizado na Figura 1A,B, medida foi feita na radiografia em perfil da coluna cervical, e repetida na $\mathrm{RM}$, em imagem ponderada em T2 no corte sagital correspondente ao centro do canal vertebral (Figura 2).

$\mathrm{O}$ índice de Torg quando menor que 0,8 representa estenose cervical absoluta, quando entre 0,8 e 1,2 traduz estenose moderada e acima ou igual a 1,2 ausência de estenose ${ }^{25,26}$

Os resultados de idade, peso e altura serão numericamente representados através de valores de média \pm desvio padrão (DP), sendo o gênero expressado em porcentagem. Comparação entre as médias obtidas de Índice de Torg serão analisadas estatisticamete através de teste $t$ de Student não-pareado, com significância dada para valores de $p<0,05$.

A análise dos dados será realizada utilizando os softwares Microsoft Office / Excel 2007 e GraphPad Prism (San Diego, CA, EUA).

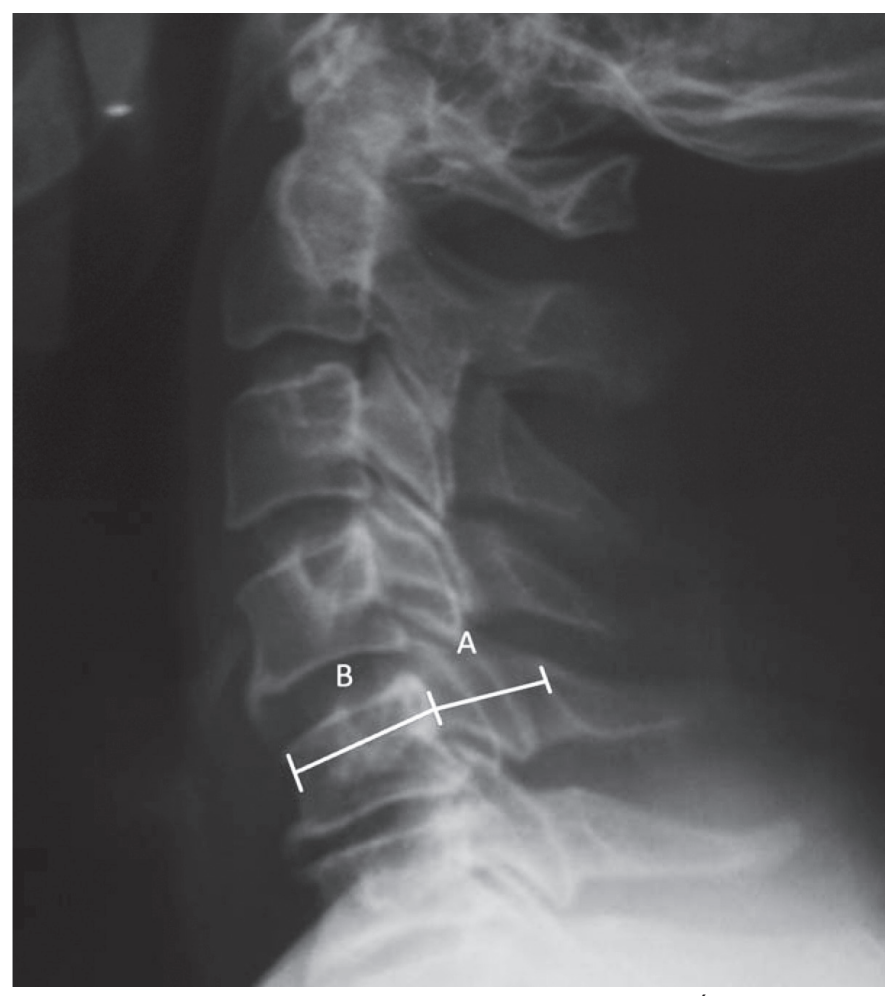

Figura 1. Esquema demonstrativo da mensuração do Índice de Torg, obtido através da relação entre a distância da superfície posterior do corpo vertebral e o ponto mais próximo à linha laminar correspondente $(A)$, dividido pelo diâmetro sagital do corpo vertebral (B). 


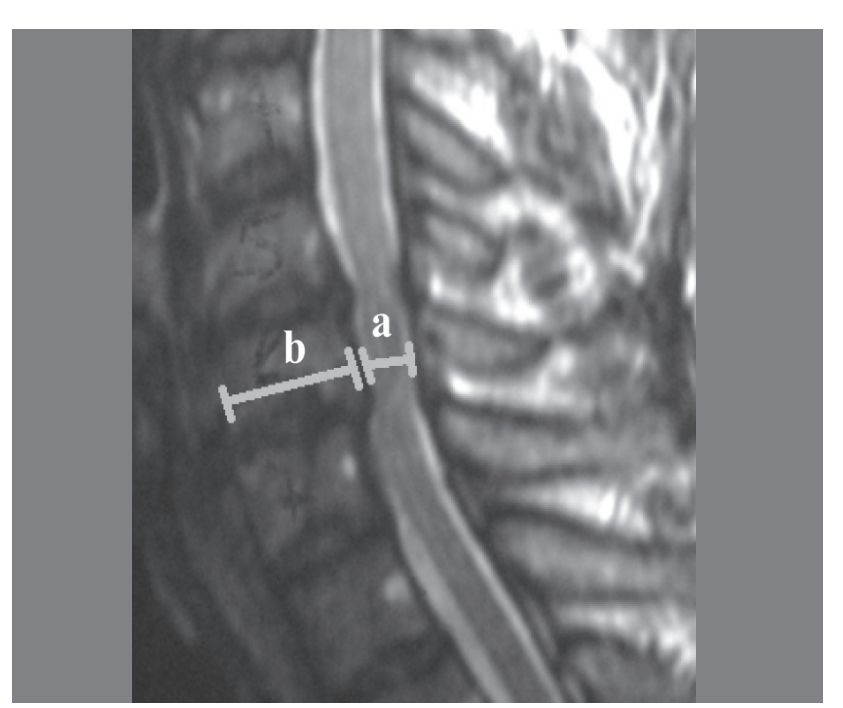

Figura 2. Medida do Índice de Torg através de Ressonância Magnética, obtido pela razão entre a distância (a) e a distância (b).

\section{RESULTADOS}

Participaram do presente estudo uma amostra de 29 pacientes com MCE, dentre eles 10 mulheres (34,5\%) e 19 homens (65,5\%), com médias de idade 48,1 \pm 11 anos, de peso 68,7 $\pm 5 \mathrm{Kg}$ e de altura 1,68 \pm 0,6 m, como pode ser verificado na Tabela 1. Houve diferença significativa entre o Índice de Torg calculado através de radiografia e RM, sendo menores os índices observados na RM (radiografia: 0,73 $\pm 0,17$ vs. RM: 0,48 $\pm 0,14, p<0,05)$. No entanto, ambas as aferições traduzem o mesmo resultado de estenose cervical absoluta (Índice de Torg $<0,8$ ) (Figura 3).

Tabela 1. Característica da amostra.

\begin{tabular}{c|c}
\hline Parâmetros & Média \pm DP \\
\hline Idade (anos) & $48,1 \pm 10,9$ \\
\hline Peso $(\mathrm{Kg})$ & $68,7 \pm 5,0$ \\
\hline Altura $(\mathrm{m})$ & $1,68 \pm 0,06$ \\
\hline
\end{tabular}

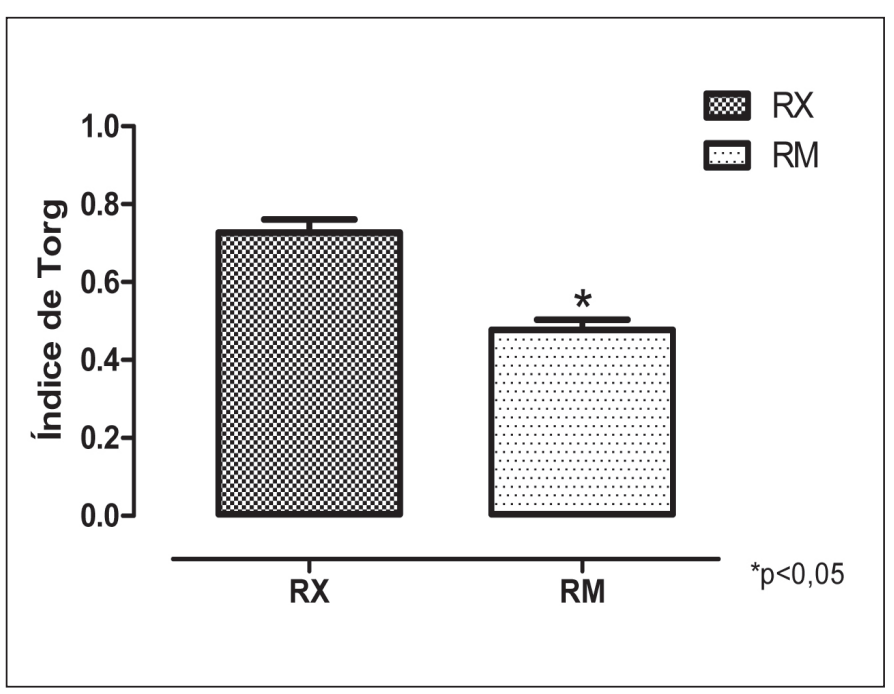

Figura 3. Comparação entre o índice de Torg obtido através de radiografia (RX) e de Ressonância Magnética (RM).

\section{DISCUSSÃO}

Nosso estudo corrobora a literatura pré-existente no que diz respeito às características dos portadores de MCE, com maior prevalência no gênero masculino e na faixa etária em torno dos 50 anos de idade ${ }^{3}$. Além disso, observamos com maior frequência a estenose cervical no nível C5, conforme relatos prévios ${ }^{3}$. Apesar de sabidamente ser mais comum na raça oriental, devido maior ossificação do ligamento longitudinal posterior, que contribui para a estenose cervical ${ }^{8}$, em nosso estudo não havia nenhum paciente da raça oriental, possivelmente por nossa região ser de imigrantes de outras etnias.

Através da radiografia em perfil dos participantes do presente estudo, obtivemos valores de Índice de Torg menores do que 0,8 , indicando que todos os pacientes possuíam uma estenose de canal cervical absoluta ${ }^{25,26}$. Esse dado foi semelhante ao encontrado na literatura mundial que considera o Índice de Torg um método diagnóstico de estenose do canal cervical $3,8,11,13-20$. O índice de Torg eliminou o viés da magnificação radiológica da medida direta do canal vertebral, o qual era causado por diferenças da distância entre o paciente, o filme e o tubo de raio-X. Este viés causava medidas não fidedignas quanto à aferição da estenose do canal cervical, como descrito no artigo em que Torg descreve o método ${ }^{6}$

Em nossa pesquisa, a radiografia demonstrou ser um bom método de diagnóstico e de screening de pacientes com MCE. Segundo Wong et al. ${ }^{8}$, a radiografia faz parte da primeira linha de investigação devido sua acessibilidade, baixo custo operacional e habilidade em mostrar a ossificação do ligamento longitudinal posterior.

Embora tenha sido encontrado o Índice de Torg com valor menor do que 0,8 em todos os pacientes do estudo, quando a medida foi realizada através de RM esse valor foi menor significativamente que o obtido na radiografia. A partir disso, sugerimos que o referido índice deve ser inicialmente medido através de radiografia como uma forma de triagem, mas ainda acreditamos na necessidade da mensuração a partir da RM, devido este exame ter se apresentado mais sensível no diagnóstico de MCE em nosso estudo.

A RM é uma ferramenta hoje utilizada em todos os pacientes com sinais e sintomas de $\mathrm{MCE}^{3,11,13}$, por confirmar o diagnóstico, bem como documentar a extensão e a gravidade da lesão ${ }^{3}$. $\mathrm{Na}$ RM visibilizamos o sinal de necrose e morte de células do sistema nervoso central conhecido como Mielomalácea. Yukawa et al. ${ }^{27}$ propuseram uma classificação de gravidade da Mielopatia Cervical através da intensidade do sinal de Mielomalácea observada na RM.

Além disso, através da RM visualizamos outras causas de estenose de canal cervical como osteófitos e hipertrofia do ligamento flavo, os quais surgem com a degeneração típica do envelhecimento, associando-se à fisiopatologia da mielopatia cervical ${ }^{28}$. Podemos observar também na RM protrusões discais, que assim como a formação osteofitária e a hipertrofia do ligamento flavo, contribuem para a estenose do canal cervical. Por sua vez, tais alterações não são claramente evidenciadas na radiografia, o que contribui para diminuir, deste modo, a sensibilidade no diagnóstico de MCE através deste exame de imagem.

\section{CONCLUSÃO}

A ressonância magnética é um exame mais fidedigno que a radiografia na aferição da estenose do canal cervical obtida através do Índice de Torg. Acreditamos, ainda, que a radiografia deve continuar sendo realizada rotineiramente nestes pacientes como método de triagem, seguida de uma melhor investigação a partir de RM. 


\section{REFERÊNCIAS}

1. Berger JR. Medical Myelopathies. Spine. 2005;5:795

2. Young WF. Cervical spondylotic myelopathy: a common cause of spinal Cord dysfunction in older persons. Am Fam Physician. 2000;62(5):1064-70, 1073.

3. Tracy JA, Bartleson JD. Cervical spondylotic myelopathy. Neurologist. 2010;16(3):176-87.

4. Crandall PH, Gregoriuis FK. Long-term follow-up of surgical treatment of cervical spondylitic myelopathy. Spine. 1977;2:139-46.

5. Orr RD, ZdeblickTA. Cervical spondylotic myelopathy. Approaches to surgical treatment. Clin Orthop Relat Res. 1999;(359):58-66.

6. Boden SD, McCowin PR, Davis DO, Dina TS, Mark AS, Wiesel S. Abnormal magneticresonance scans of the cervical spine in asymptomatic subjects. A prospective investigation. J Bone Joint Surg Am. 1990;72(8):1178-84.

7. Inamasu J, Guiot BH, Sachs DC. Ossification of the posterior longitudinal ligament: an update on its biology, epidemiology, and natural history. Neurosurgery. 2006;58(6):1027-39.

8. Wong TM, Leung HB, Wong WC. Correlation between magnetic resonance imaging and radiographic measurement of cervical spine in cervical myelopathic patients. J Orthop Surg (Hong Kong). 2004;12(2):239-42.

9. Lunsford LD, Bissonette DJ, Zorub DS. Anterior surgery for cervical disc disease. Part 2: Treatment of cervical spondylotic myelopathy in 32 cases. J Neurosurg. 1980;53(1):12-9.

10. Hukuda S, Mochizuki T, Ogata M, Shichikawa K, Shimomura Y. Operations for cervical spondylotic myelopathy. A comparison of the results of anterior and posterior procedures. J Bone Joint Surg Br. 1985;67(4):609-15.

11. Prasad SS, O'Malley M, Caplan M, Shackleford IM, Pydisetty RK. MRI measurements of the cervical spine and their correlation to Pavlov's ratio. Spine (Phila Pa 1976). 2003;28(12):1263-8.

12. Boijsen E. The cervical spinal canal in intraspinal expansive processes. Acta Radiol. 1954;42(2): 101-15.

13. Tierney RT, Maldjian C, Mattacola CG, Straub SJ, Sitler MR. Cervical Spine Stenosis Measures in Normal Subjects. J Athl Train. 2002;37(2):190-193.

14. Torg JS. Cervical spinal stenosis with cord neurapraxia: evaluations and decisions regarding participation in athletics. Curr Sports Med Rep. 2002;1(1):43-6.

15. Yue WM, Tan SB, Tan MH, Koh DC, Tan CT. The Torg-Pavlov ratio in cervical spondylotic myelopathy: a comparative study between patients with cervical spondylotic myelopathy and a nonspondylotic, nonmyelopathic population. Spine (Phila Pa 1976). 2001;26(16):1760-4.
16. Giovanini MA, Day AL. Spinal Injuries in athletes with cervical stenosis. Techn Neurosurg. 1999:5(2):185-93.

17. Keny SM, Suh SW, Song HR, Vaidya SV, Machavarapu MM. Morphometric determinants of the sagittal dimensions of the cervical spinal canal in achondroplasia: an analysis of the reliability of the Torg ratio. J Spinal Disord Tech. 2006;19(7):523-7.

18. Boockvar JA, Durham SR, Sun PP. Cervical spinal stenosis and sports-related cervical cord neurapraxia in children. Spine (Phila Pa 1976). 2001;26(24):2709-12.

19. Herzog RJ, Wiens JJ, Dillingham MF, Sontag MJ. Normal cervical spine morphometry and cervical spinal stenosis in asymptomatic professional football players. Plain film radiography, multiplanar computed tomography, and magnetic resonance imaging. Spine (Phila Pa 1976). 1991;16(6 Suppl):S178-86.

20. Karakas P, Bozkir MG. Reference values for radiologic evaluation of cervical canal, vertebral body, and Torg-Pavlov ratio. Neurosurg Quarterly. 2007;17(4):291-293

21. Blackley HR, Plank LD, Robertson PA. Determining the sagittal dimensions of the canal of the cervical spine. The reliability of ratios of anatomical measurements. J Bone Joint Surg Br. 1999;81(1):110-2.

22. Moskovich R, Shott $\mathrm{S}$, Zhang ZH. Does the cervical canal to body ratio predict spinal stenosis? Bull Hosp Jt Dis. 1996;55(2):61-71.

23. Ryu JS, Chae JW, Cho WJ, Chang H, Moon MS, Kim SS. Cervical myelopathy due to single level prolapsed disc and spondylosis: a comparative study on outcome between two groups. Int Orthop. 2010;34(7):1011-5.

24. Kim YJ, Oh SH, Yi HJ, Kim YS, Ko Y, Oh SJ. Myelopathy caused by soft cervical disc herniation : surgical results and prognostic factors. J Korean Neurosurg Soc. 2007;42(6):441-5.

25. Pavlov H, Torg JS, Robie B, Jahre C. Cervical spinal stenosis: determination with vertebral body ratio method. Radiology. 1987;164(3):771-5.

26. Torg JS, Pavlov H, Genuario SE, Sennett B, Wisneski RJ, Robie BH, Jahre C. Neurapraxia of the cervical spinal cord with transient quadriplegia. J Bone Joint Surg Am. 1986;68(9):1354-70.

27. Yukawa $Y$, Kato $F$, Yoshihara $H$, Yanase $M$, Ito K. MR T2 image classification in cervical compression myelopathy: predictor of surgical outcomes. Spine (Phila Pa 1976). 2007;32(15):1675-8.

28. Suk KS, Kim KT, Lee JH, Lee SH, Kim JS, Kim JY. Reevaluation of the Pavlov ratio in patients with cervical myelopathy. Clin Orthop Surg. 2009;1(1):6-10. 